\title{
Perfil clínico de sensibilización a hongos en Medellín, Colombia
}

Ingrid Bissinger, ${ }^{1}$ José Bareño ${ }^{2}$

\section{Resumen}

ANTECEDENTES: la sensibilización a hongos se relaciona con enfermedades alérgicas como: asma, rinitis, conjuntivitis y dermatitis. En general, se asocia con sensibilidad a otros alérgenos. El patrón multisistémico se asoció en Medellín con la sensibilización a hongos.

OBJETIVO: determinar el perfil clínico de la sensibilización a hongos en la ciudad de Medellín, Colombia.

MATERIAL Y MÉTODO: estudio observacional, descriptivo y retrospectivo consistente en la revisión de historias clínicas de pacientes menores de 70 años de edad con sensibilidad a hongos en las pruebas de neumoalergenos realizadas entre el 1 enero de 2011 y el 31 de marzo de 2014 en la consulta de alergología de dos clínicas. Se evaluaron 16 hongos y la coexistencia de enfermedades alérgicas y las condiciones ambientales.

RESULTADOS: de 897 pruebas de prick realizadas, $12.8 \%$ resultaron positivas a alguno de los hongos, el más frecuente fue Candida albicans con $30.4 \%$. La rinitis se encontró en $100 \%$ de los pacientes, asma en $46.1 \%$, conjuntivitis en $92.2 \%$ y el patrón mutisistémico en $9.6 \%$. El patrón mutisistémico se asoció con ser joven con riesgo de 9.259, IC 95\%: 1.14-74.95 y a estar sensibilizado a Aspergillus fumigatus con un riesgo de 4.381, IC 95\%: 1.116-17.204.

CONCLUSIONES: el patrón de sensibilidad mostró mayor sensibilidad a Candida. La mayoría de los pacientes está polisensibilizada. El patrón multisistémico es más frecuente en niños, se relaciona con sensibilidad a Aspergillus. Por los hallazgos de este estudio, alérgenos como Candida y Cladosporium deberían evaluarse en Medellín.

PALABRAS CLAVE: hongos, asma, rinitis, patrón multisistémico, sensibilización.

Rev Alerg Méx 2016 Apr-Jun;63(2):123-134.

\section{Clinical profile of sensitization to fungi in Medellin, Colombia}

Ingrid Bissinger, ${ }^{1}$ José Bareño

\begin{abstract}
BACKGROUND: Fungi sensitization correlates with patologies like asthma, rhinitis, conjunctivitis and dermatitis. In general it is associated with other sensitizations. In Medellin the multisystemic pattern is associated with fungi sensitization.
\end{abstract}

OBJECTIVE: To determine the clinical pattern of the fungi sensitization in Medellin.

\author{
${ }^{1}$ El Rosario Sede Tesoro, Clínica Conquistadores, \\ Medellín, Antioquia, Colombia \\ ${ }^{2}$ Universidad CES, Medellín, Antioquia, Colombia \\ Recibido: 17 de junio 2015 \\ Aceptado: 24 de febrero 2016 \\ Correspondencia \\ Ingrid Bissinger \\ ingridbissingern@yahoo.com
}

Este artículo debe citarse como

Bissinger I, Bareño J. Perfil clínico de sensibilización a hongos en Medellín, Colombia. Rev Alerg Méx. 2016 abr-jun;63(2):123-134. 
MATERIAL AND METHOD: We reviewed the medical records of 897 patients younger than 70 years old with fungi sensitization in the prick test during the period 1 January 2011 to 31 March 2014 in the allergy facility from 2 clinics. We evaluate 16 fungi and the presence of allergic diseases as well as the environmental conditions.

RESULTS: Form 897 prick test, $12.8 \%$ were positive to fungi, and the most frequent was Candida albicans with $30.4 \%$. Rhinitis were present in $100 \%$ of patients, asthma in $46.1 \%$, conjunctivitis in $92.2 \%$ and the multisystemic pattern in $9.6 \%$. The multisystemic pattern was associated with younger age with a risk of 9,259, 95\% Cl: 1.14-74.95 and with Aspergillus fumigatus sensitivity with a risk of $4.381,95 \% \mathrm{Cl}$ : $1,116-17,204$

CONCLUSIONS: The pattern of sensitivity was higher with Candida albicans. Most patients were polysensitized. The multisystemic pattern was associated with been younger and with Aspergillus fumigatus sensitivity. From the findings of this study, allergens like Candida and Aspergillus fumigatus should be tested in Medellin.

KEY WORDS: Fungi, asthma, rhinitis, multisystemic pattern.

\author{
${ }^{1}$ El Rosario Sede Tesoro, Clínica Conquista- \\ dores, Medellín, Antioquia, Colombia \\ 2 Universidad CES, Medellín, Antioquia, \\ Colombia
}

Correspondence

Ingrid Bissinger

ingridbissingern@yahoo.com

\section{ANTECEDENTES}

Se reconoce que los hongos perticipan en los padecimientos alérgicos y no alérgicos. Está demostrado que los hongos pueden producir alergia tipo I en individuos susceptibles; los identificados con mayor frecuencia son ascomicetos, basidiomicetos y deuteromicetos. ${ }^{1}$ Los hongos se encuentran en el interior de las viviendas y en espacios exteriores. La concentración de hongos en los interiores se ha asociado con diversos factores, entre los más relevantes están la humedad visible, las plantas y las mascotas. ${ }^{2-5}$

La humedad y los hongos visibles en las viviendas se han asociado con sensibilización a éstos y a enfermedades alérgicas como: asma, rinitis y dermatitis, así como con la severidad de éstas. ${ }^{6-10}$ Son escasos los estudios que no identifican esta asociación. ${ }^{11,12}$

La sensibilidad a hongos en pacientes alérgicos se ha reportado en numerosos estudios y así se ha determinado que en pacientes con rinitis y asma varía de 3 a 20\% en Europa, 10\% en Escandinavia, de 30 a $60 \%$ en Estados Unidos ${ }^{13}$ y $6.7 \%$ en China. ${ }^{14}$ La expresión clínica presentada por pacientes sensibilizados a hongos es: asma, dermatitis, rinitis. La sensibilidad a hongos se asocia con sensibilidad a otros alérgenos. ${ }^{8}$ Está demostrada la asociación entre la sensibilidad a hongos y la severidad del asma ${ }^{15-18}$ y de los síntomas de rinitis. ${ }^{19}$

Existe relación entre la sensibilidad a hongos y la edad; es más frecuente en niños y jóvenes que en adultos y en ancianos. ${ }^{9,20-22}$ En Monterrey, México, se encontró mayor prevalencia de sensibilidad a hongos en el grupo de 0 a 10 años. ${ }^{23}$

En Colombia se demostró que la prevalencia de síntomas de asma y rinitis va en aumento (asma de 10.4 a $12 \%$ y rinitis 22.6 a $32 \%$ ). ${ }^{24,25} \mathrm{El}$ estudio FRAAT de Cartagena demostró que 3\% de la población estudiada tenía sensibilidad al menos a un hongo evaluado (Alternaria, Asper- 
gillus, Penicillium) y $100 \%$ de los sensibilizados a hongos tenían síntomas de alergia. Un estudio efectuado en Bucaramanga demostró asociación entre los mohos dentro de la vivienda y las sibilancias respiratorias compatibles con asma. ${ }^{26}$ Otro estudio llevado a cabo en la misma ciudad encontró que las viviendas tenían diferentes hongos, aislaron Cladosporium sp en 98\%, Fusarium sp en $82 \%$, Aspergillus sp en $54 \%$, Penicillium en $49 \%$, Acremonium sp $7.82 \%$. Este estudio demostró asociación entre síntomas de asma y el aislamiento de Acremonium sp, pero no con los otros hongos. ${ }^{27}$

Existen muy pocos estudios en Colombia en donde se evalúe el efecto de la existencia de hongos en las viviendas o en los sitios de trabajo. En el municipio del César la humedad se asoció con mayor frecuencia de reporte de tos seca nocturna, a sibilancias en el último año, sibilancias nocturnas, dermatitis atópica y ausentismo escolar. ${ }^{28}$

En el estudio realizado por Sánchez-Caraballo en la ciudad de Medellín se encontró que el patrón multisistémico definido como la coexistencia simultánea de asma, rinitis y dermatitis se asociaba con la sensibilidad a hongos. La sensibilidad en orden de frecuencia fue a Alternaria alternata, Aspergillus fumigatus, Candida albicans, Cladosporium herbarum y Tricophyton mentagrophytes. ${ }^{29}$

Esta prevalencia baja de sensibilidad a hongos encontrada en Medellín es similar a la encontrada en pacientes con asma o rinitis en el mundo $8,13,14$ y se puede presentar aislada o en racimos con sensibilidad a varios hongos. Raras veces se encuentra sensibilización a hongos aislada; es más frecuente la sensibilidad concomitante con otros alérgenos. ${ }^{9}$

En Colombia se desconoce el patrón de sensibilización a múltiples hongos y su expresión clínica, al igual que la asociación con otros alérgenos, quizá debido a que la mayor parte de los alergólogos en Colombia evalúa pocos hongos durante las pruebas de prick con neumoalérgenos. En general, se evalúan en Colombia solo tres hongos, Alternaria, Aspergillus y Cladosporium y muy pocos alergólogos incluyen un cuarto o un quinto hongo. Este estudio pretende determinar cuál es el patrón clínico de los pacientes sensibilizados a hongos en la ciudad de Medellín y determinar si existe sensibilidad concomitante con otros alérgenos y si el patrón mutisistémico se asocia con esta sensibilidad a hongos.

\section{MATERIAL Y MÉTODO}

Estudio observacional, descriptivo y retrospectivo. Se evaluaron las pruebas de prick a neumoalérgenos realizadas entre el 1 enero de 2011 y el 31 de marzo de 2014 en la consulta de alergología de la Clínica el Rosario, sede Tesoro y Clínica Conquistadores de la ciudad de Medellín. Se incluyeron pacientes menores de 70 años con historia completa efectuado por un alergólogo y con resultados positivos al menos a un hongo, independientemente de si existían o no sensibilidades a otros alérgenos.

Se consideraron positivas las pruebas con pápulas mayores de $3 \mathrm{~mm}$ en promedio tomando el diámetro mayor y el ortogonal (diámetro mayor más el diámetro ortogonal dividido entre 2). Se tomó como control positivo la histamina y como control negativo la solución salina. Los alérgenos evaluados con extractos estandarizados de Laboratorios Leti (Madrid, España) fueron: Dermatophagoides pteronyssinus, Dermatophagoides farinae, Blomia tropicalis, cucaracha (Periplaneta americana), perro, gato, caballo, mosquito, roble, abedul, ciprés, pólenes de cereales (avena, cebada, centeno, trigo, maíz), pólenes de gramíneas (Dactylis, Festuca, Lollium perenne, Phleum pratense, Poa, Cynodon dactylon, Phragmitis communis), pólenes de hierbas 
(Artemisa, Chenopodium, Plantago lanceolata, Salsola kali, Parietaria judaica, Taraxacum, Ambrosia elatior) y los siguientes hongos: Alternaria alternata, Aspergillus fumigatus, Cladosporium herbarum, Mucor mucedo, Penicillium, Candida albicans, Aspergillus niger, Curvularia lunata, Fusarium, Botrytis, Stemphylium, Rhizopus nigricans, Thricophytum mentagrophites, Aureubasidium, Helmintosporium y Phoma.

Se evaluaron las concentraciones de inmunogobulina E sérica total y el valor de eosinófilos en sangre periférica en los pacientes con reporte en la historia clínica de alergología.

Las enfermedades incluidas fueron: asma según criterios GINA, rinitis según criterios ARIA, conjuntivitis (incluidas queratoconjuntivitis vernal, queratoconjuntivitis atópica, conjuntivitis de papilas gigante, conjuntivitis estacional y perenne), dermatitis atópica según criterios de Hanifin Rajka (no se incluye el score). El diagnóstico se estableció en el momento de la consulta. Simultáneamente se evaluaron la coexistencia o el antecedente de dermatitis de contacto, urticaria y angioedema, alergia a alimentos y alergia a medicamentos en cada paciente para dar el perfil clínico de quienes estaban sensibilizados a hongos. No se analizaron padecimientos diferentes a la alergia que pudieran estar relacionados con hongos, como las infecciones o las toxicidades. Se evaluaron los antecedentes familiares de enfermedades alérgicas en familiares de hasta segundo grado al igual que en los pacientes. El análisis de las condiciones ambientales incluyó la coexistencia de mascotas en el hogar, al igual que las humedades actuales y previas en el hogar o sitios de trabajo, la presencia de tapetes, el consumo de cigarrillos por parte del paciente o por segunda mano (fumador pasivo). La información de los menores de edad fue suministrada por los padres o tutores del menor.
Ética

Este estudio se rigió en sus aspectos éticos por la Declaración de Helsinki, adoptada por la Asamblea Médica de Helsinki en 1964 y revisada por la XXIX Asamblea Mundial Médica en Tokio, Japón, en 1975.

\section{Análisis estadístico}

Los análisis se efectuaron con el programa IBM_SPSS versión 21 para Windows, licencia de la Universidad CES. Las características generales de la población para las variables cualitativas se presentaron con frecuencias y porcentajes y para las variables cuantitativas se realizó el análisis de normalidad mediante las pruebas de ShapiroWilk y Kolmogorow-Smirnow. La asociación entre variables cualitativas se determinó por el estadístico de $X^{2}$. Para determinar la relación entre variables cuantitativas se realizó correlación de Pearson y Spearman. Para determinar la diferencia de promedios entre grupos se trabajó con el estadístico t de Student o $U$ de Mann Whitney según la distribución de la variable cuantitativa. Se estableció como significativo un valor de $\mathrm{p}<0.05$.

\section{RESULTADOS}

\section{Características generales de la población}

Se analizaron 897 pruebas de prick de neumoalérgenos del servicio de Alergología de la Clínica El Rosario el Tesoro y de la Clínica Conquistadores de la ciudad de Medellín, Colombia, del 1 de enero de 2011 al 31 de marzo de 2014. De estos registros, 115 cumplieron los criterios establecidos y se encontró una sensibilización a hongos en la población de 12.8\%. De los 115 pacientes, $58.2 \%$ eran mujeres y $41.7 \%$ hombres. La edad tuvo una distribución no normal con una mediana de 15 años y rango intercuartil de 61. 
El 50\% de la muestra tenía 15 o menos años. Se dividió la muestra en niños ( $\leq 17$ años) y adultos ( $\geq 18$ años) para poder establecer diferencias entre grupos; se encontraron 64 niños $(55.6 \%)$ y 51 adultos (44.5\%). Los niños tenían una distribución normal de la edad con promedio de edad de 10.6 con $\mathrm{DE}=3.686$ y los adultos una distribución no normal con una mediana de 30 años con un rango intercuartil de 15 .

\section{Enfermedades alérgicas}

Del total de la población de pacientes sensibilizados a hongos, el $100 \%$ tenían rinitis, 92.2\% conjuntivitis, $46.1 \%$ asma, 20\% dermatitis atópica, $16.5 \%$ alergia a alimentos, $14.8 \%$ alergia a medicamentos, $4.3 \%$ dermatitis de contacto, $4.3 \%$ urticaria crónica y $22.6 \%$ tenían antecedente de urticaria aguda. El patrón multisistémico definido como la coexistencia de rinitis, conjuntivitis, asma y dermatitis atópica se encontró en 9.6\% de los pacientes (Cuadro 1).

Al comparar los dos grupos de edad se encontró que los adultos eran $72.5 \%$ mujeres, $90.2 \%$ tenían conjuntivitis, el $100 \%$ tenía rinitis y de

Cuadro 1. Prevalencia de enfermedades alérgicas en pacientes sensibilizados a hongos

\begin{tabular}{lccc}
\hline & n & & Porcentaje \\
Distribución por edad & Niños & 64 & 55.65 \\
& Adultos & 51 & 44.35 \\
Sexo & Masculino & 48 & 41.74 \\
& Femenino & 67 & 58.26 \\
Rinitis & 115 & & 100 \\
Conjuntivitis & 106 & 92.2 \\
Asma & 53 & 46.1 \\
Urticaria aguda & 26 & 22.6 \\
Dermatitis atópica & 23 & 20 \\
Alergia a alimentos & 19 & 16.5 \\
Alergia a medicamentos & 17 & 14.8 \\
Dermatitis de contacto & 5 & 4.3 \\
Urticaria crónica & 5 & 4.3 \\
Patrón multisistémico & 11 & 9.6
\end{tabular}

ésta $78.4 \%$ era persistente moderada a grave. El $25.5 \%$ de los adultos tenía asma; $9.8 \%$ dermatitis atópica; $5.9 \%$ dermatitis de contacto; $21.6 \%$ alergia a alimentos; $11.8 \%$ alergia a medicamentos; urticaria crónica $7.8 \%$ y antecedente de urticaria aguda $21.6 \%$. En los niños, $46.9 \%$ eran mujeres, $93.8 \%$ tenían conjuntivitis, el $100 \%$ tenía rinitis y de ésta $93.8 \%$ era persistente moderada a grave. El $62 \%$ de los niños tenían asma, $28.1 \%$ dermatitis atópica, $3.1 \%$ dermatitis de contacto, $12.5 \%$ alergia a alimentos, $17.2 \%$ alergia a medicamentos, $1.6 \%$ urticaria crónica y $23.4 \%$ antecedente de urticaria aguda (Cuadro 2).

Los hallazgos muestran que los niños tenían más rinitis persistente moderada a grave, más asma, más dermatitis atópica, más alergia a medicamentos y más urticaria aguda que los adultos, mientras estos últimos eran más mujeres, tenían más alergia a alimentos y más urticaria crónica. Estas relaciones fueron estadísticamente significativas solo para edad y asma, edad y dermatitis atópica y edad y patrón multisistémico. La edad se relacionó con asma con $\chi^{2}=15.646$

Cuadro 2. Prevalencia de enfermedades alérgicas por grupos

\begin{tabular}{|c|c|c|c|c|c|}
\hline \multirow[b]{2}{*}{ Sexo } & \multicolumn{2}{|c|}{ Niños } & \multicolumn{2}{|c|}{ Adultos } & \multirow{2}{*}{$\begin{array}{c}\text { Nivel de } \\
\text { significación }\end{array}$} \\
\hline & $\mathrm{n}$ & $\%$ & $\mathrm{n}$ & $\%$ & \\
\hline Masculino & 34 & 53.1 & 14 & 27.5 & \\
\hline Femenino & 30 & 46.9 & 37 & 72.5 & \\
\hline $\begin{array}{l}\text { Rinitis persistente } \\
\text { moderada a grave }\end{array}$ & 60 & 93.8 & 40 & 78.4 & NS \\
\hline Conjuntivitis & 60 & 93.8 & 46 & 90.2 & NS \\
\hline Asma & 40 & 62.5 & 13 & 25.5 & $p=0.000076$ \\
\hline Urticaria aguda & 15 & 23.4 & 11 & 21.6 & NS \\
\hline Dermatitis atópica & 18 & 28.1 & 5 & 9.8 & $P=0.015$ \\
\hline Alergia a alimentos & 8 & 12.5 & 11 & 21.6 & NS \\
\hline $\begin{array}{l}\text { Alergia a medica- } \\
\text { mentos }\end{array}$ & 11 & 17.2 & 6 & 11.8 & NS \\
\hline $\begin{array}{l}\text { Dermatitis de } \\
\text { contacto }\end{array}$ & 2 & 3.1 & 3 & 5.9 & NS \\
\hline Urticaria crónica & 1 & 1.6 & 4 & 7.8 & NS \\
\hline $\begin{array}{l}\text { Patrón multisisté- } \\
\text { mico }\end{array}$ & 10 & 15.63 & 1 & 1.96 & $p=0.013$ \\
\hline
\end{tabular}


$\mathrm{p}=0.000076$, riesgo 4.872 , IC $95 \%=2.171$ 10.928. La edad también se relacionó con la dermatitis atópica con $\chi^{2}=5.954(p=0.015)$, riesgo 3.6, IC 95\% = 1.233-10.514. La edad se relacionó con el patrón multisistémico con $\chi^{2}=$ $6,126(\mathrm{p}=0.013)$ y con un riesgo de 9.259, IC $95 \%=1.14-74.95$ (Cuadro 2).

A pesar de que el patrón multisistémico fue más frecuente en hombres que en mujeres, la diferencia no fue estadísticamente significativa. Este patrón multisistémico no se relacionó con la sensibilidad a ácaros, ni a epitelios ni a pólenes de árboles, cereales, gramíneas ni a hierbas pero se relacionó con sensibilidad a Aspergillus fumigatus $\operatorname{con} \chi^{2}=5.119(\mathrm{p}=0.024)$ con un riesgo de 4.381 , IC $95 \%=1.116-17.204$.

Al analizar a los pacientes con o sin patrón multisistémico se encontró que quienes tenían el patrón tenían humedad actual en 18.2 vs $9.8 \%$, humedad previa 18.2 vs $13.8 \%$, tenían tapetes 9.1 vs $13.2 \%$, no fumaban el 100 vs $94.6 \%$, eran fumadores pasivos 9.1 vs $6.7 \%$ y tenían mascota 27.3 vs 36\% (Cuadro 3). Este patrón multisistémico en la población general no se asoció con humedad previa $\left(\chi^{2}=0.160\right.$, $\mathrm{p}=0.689) \mathrm{ni}$ con humedad actual $\left(\chi^{2}=0.778\right.$, $\mathrm{p}=0.378) \mathrm{ni}$ con tener tapetes $\left(\chi^{2}=0.155\right.$, $\mathrm{p}=0,694)$ ni con fumar $\left(\chi^{2}=0.559, \mathrm{p}=0.455\right) \mathrm{ni}$ con ser fumador pasivo $\left(\chi^{2}=0.092, p=0.762\right)$ ni con tener mascotas $\left(\chi^{2}=0.341, p=0.559\right)$.

Cuadro 3. Condiciones ambientales según la existencia o no del patrón multisistémico

\begin{tabular}{lcc}
\hline Condición & $\begin{array}{c}\text { Con patrón mul- } \\
\text { tisistémico } \mathbf{( \% )}\end{array}$ & $\begin{array}{c}\text { Sin patrón multi- } \\
\text { sistémico } \mathbf{( \% )}\end{array}$ \\
\hline Humedad actual & 18.2 & 9.8 \\
Humedad previa & 18.2 & 13.8 \\
Con tapetes & 9.1 & 13.2 \\
No fumador & 100 & 94.6 \\
Fumador pasivo & 9.1 & 6.7 \\
Con mascotas & 27.3 & 36
\end{tabular}

Al comparar a los niños con los adultos no se encontró asociación entre el patrón multisistémico y estas variables ambientales en cada uno de los grupos.

Los pacientes con asma (niños y adultos) tenían humedad actual $11.5 \%$, humedad previa $15.4 \%$, tapetes $15.4 \%$, fumaban solo $3.8 \%$, eran fumadores pasivos $11.3 \%$ y tenían mascota $37.7 \%$. Los que no tenían asma tenían humedad actual $9.8 \%$, humedad previa $13.6 \%$, tapetes $11.9 \%$, fumaban $4.8 \%$, eran fumadores pasivos 4.9 , y tenían mascota $34.4 \%$.

En la población general no hubo relación entre asma y humedad actual $\left(\chi^{2}=0.114 \mathrm{p}=0.736\right) \mathrm{ni}$ con humedad previa $\left(\chi^{2}=0.099, \mathrm{p}=0.753\right) \mathrm{ni}$ con tener tapetes $\left(\chi^{2}=0.397, \mathrm{p}=0.529\right) \mathrm{ni}$ con fumar $\left(\chi^{2}=0.083, p=0.773\right)$ ni con ser fumador pasivo $\left(\chi^{2}=2.37, \mathrm{p}=0.124\right)$ ni con tener mascota $\left(\chi^{2}=0.177, p=0.674\right)$

El valor de la inmunoglobulina E sérica total se reportó en 71 pacientes $(61.7 \%$ de la población) con mediana de 561 y rango intercuartil de 1145 y con $75 \%$ de los valores por debajo de 1412. Al evaluar por separado los grupos se encontraron valores de IgE en $76.6 \%$ de los niños y en $43.1 \%$ de los adultos. En los niños la mediana del valor de IgE fue de 742 con rango intercuartil de 1011.55 mientras que en los adultos la mediana fue de 236 con rango intercuartil de 265.78. U de Mann-Whitney Z $=-4.663, p=0.000$

El valor de eosinofilos en sangre periférica se reportó en 67 pacientes (58.2\%) con media de 599.96. Se obtuvieron valores de eosinófilos en sangre periférica en $68.8 \%$ de los niños (44 niños) y en $45.1 \%$ de los adultos (23 adultos). En los niños la mediana fue de 577 con rango intercuartil de 575 y en los adultos la mediana fue de 190 con rango intercuartil de 390. $U$ de Mann Whitney $Z=-3,427, p=0.001$ 


\section{Condiciones ambientales}

Se encontró que del total de los pacientes sensibilizados a hongos $10.6 \%$ tenían humedad actual, $14.4 \%$ humedad previa, $35.6 \%$ tenían mascota, $13.5 \%$ tenían tapetes en sus viviendas, $4.4 \%$ era fumador activo y $7.9 \%$ era fumador pasivo. Las condiciones ambientales fueron similares entre los grupos (niños y adultos) (Cuadro 4).

\section{Antecedentes familiares de alergia}

Los pacientes del estudio tenían antecedentes familiares de asma en $53.9 \%$, de rinitis en $74.8 \%$, de dermatitis atópica $11.3 \%$, de dermatitis de contacto $6.1 \%$, de alergia a alimentos $10.4 \%$ y alergia a medicamentos $23.5 \%$. Las frecuencias de estos antecedentes en niños y adultos se anotan en el Cuadro 4.

\section{Sensibilización}

En la población estudiada se encontró que la sensibilidad a hongos era más frecuente por Can-

Cuadro 4. Condiciones ambientales y antecedentes familiares en población total (niños y adultos)

\begin{tabular}{|c|c|c|c|}
\hline Condición & $\begin{array}{l}\text { Población } \\
\text { total }(\%)\end{array}$ & $\begin{array}{l}\text { Niños } \\
(\%)\end{array}$ & $\begin{array}{c}\text { Adultos } \\
(\%)\end{array}$ \\
\hline Humedad actual & 10.6 & 9.4 & 12.2 \\
\hline Humedad previa & 14.4 & 15.9 & 12.5 \\
\hline Con tapetes & 13.5 & 14.3 & 12.5 \\
\hline Fumador & 4.4 & 0 & 10 \\
\hline Fumador pasivo & 7.9 & 6.3 & 10 \\
\hline Con mascotas & 35.6 & 40.6 & 30 \\
\hline $\begin{array}{l}\text { Antecedente familiar de } \\
\text { asma }\end{array}$ & 53.9 & 70.3 & 33.3 \\
\hline $\begin{array}{l}\text { Antecedente familiar de } \\
\text { rinitis }\end{array}$ & 74.8 & 84.4 & 62.7 \\
\hline $\begin{array}{l}\text { Antecedente familiar de } \\
\text { dermatitis atópica }\end{array}$ & 11.3 & 12.5 & 9.8 \\
\hline $\begin{array}{l}\text { Antecedente familiar de } \\
\text { dermatitis de contacto }\end{array}$ & 6.1 & 4.7 & 7.8 \\
\hline $\begin{array}{l}\text { Antecedente familiar de } \\
\text { alergia a alimentos }\end{array}$ & 10.4 & 12.5 & 7.8 \\
\hline $\begin{array}{l}\text { Antecedente familiar de } \\
\text { alergia a medicamentos }\end{array}$ & 23.5 & 21.9 & 25.5 \\
\hline
\end{tabular}

dida con $30.4 \%$ seguida por la sensibilidad en $28.7 \%$ a Cladosporium, $21.7 \%$ a Stemphilium, $20 \%$ a Botrytis, $20 \%$ a Trichophyton, $18.2 \%$ a Alternaria, $18.2 \%$ a Curvularia, $17.3 \%$ a Fusarium, $15.6 \%$ a Mucor, $15.6 \%$ a Rhizopus, $14.7 \%$ a Aureubasidium, $13.9 \%$ a Aspergillus niger, $13.04 \%$ a Aspergillus, $7.8 \%$ a Penicillium, $4.3 \%$ a Helmintosporium, $2.6 \%$ a Phoma. Los pacientes estaban sensibilizados a un hongo en $52.2 \%$, sensibilizados a dos hongos en $15.7 \%$ y a más de tres hongos en $32.2 \%$ de los casos. El tamaño de las pápulas fue, en general, pequeño, cercano al límite de positividad (Cuadro 5). Los porcentajes de positividad a cada hongo evaluado en niños y en adultos se muestran en el Cuadro 6.

Al comparar la mediana o media (según aplique) del tamaño de las pápulas de los diferentes hongos entre niños y adultos se encontró solo diferencia estadísticamente significativa en Aspergillus fumigatus. $U$ de Mann Whitney con $\mathrm{p}=0.026$ (niños media 3.5 y DE $=0.75$ vs adulto

Cuadro 5. Porcentajes de sensibilidad a hongos. Tamaño de las pápulas

\begin{tabular}{|c|c|c|c|}
\hline Hongos & $\begin{array}{l}\text { Porcen- } \\
\text { taje } \\
\text { positivo }\end{array}$ & $\begin{array}{l}\text { Mediana } \\
\text { del diá- } \\
\text { metro de } \\
\text { pápulas } \\
(\mathbf{m m})\end{array}$ & $\begin{array}{c}\text { Rango in- } \\
\text { tercuartil } \\
\text { tamaño de } \\
\text { pápulas }\end{array}$ \\
\hline Candida & 30.43 & 4.0 & 1.0 \\
\hline Cladosporium & 28.7 & 4.0 & 1.0 \\
\hline Stemphilium & 21.7 & 4.0 & 1.3 \\
\hline Botrytis & 20 & 4.0 & 0.5 \\
\hline Trichophyton & 20 & 4.0 & 2.5 \\
\hline Alternaria & 18.3 & 4.5 & 0.8 \\
\hline Curvularia & 18.3 & 3.5 & 1.5 \\
\hline Fusarium & 17.4 & 3.5 & 1.3 \\
\hline Mucor & 15.7 & 4.0 & 1.1 \\
\hline Rhizopus & 15.6 & 4.0 & 1.0 \\
\hline & & \multicolumn{2}{|c|}{ Media \pm DE } \\
\hline Aureubasidium & 14.8 & \multicolumn{2}{|c|}{$4.09 \pm 1.04$} \\
\hline Aspergillus niger & 13.9 & \multicolumn{2}{|c|}{$4.0 \pm 0.8$} \\
\hline Aspergillus fumigatus & 13.9 & \multicolumn{2}{|c|}{$3.81 \pm 0.77$} \\
\hline Penicillium & 7.8 & \multicolumn{2}{|c|}{$4.44 \pm 1.16$} \\
\hline Helmintosporium & 4.35 & \multicolumn{2}{|c|}{$4.7 \pm 0.57$} \\
\hline Phoma & 2.65 & \multicolumn{2}{|c|}{$4.17 \pm 1.61$} \\
\hline
\end{tabular}


Cuadro 6. Porcentajes de positividad a los diferentes hongos en población total. Niños y adultos

\begin{tabular}{lccc}
\hline Hongos & $\begin{array}{c}\text { Porcentaje } \\
\text { positivo }\end{array}$ & $\begin{array}{c}\text { Porcentaje } \\
\text { positivo } \\
\text { de niños }\end{array}$ & $\begin{array}{c}\text { Porcentaje } \\
\text { positivo de } \\
\text { adultos }\end{array}$ \\
Candida & 30.43 & 25 & 37.3 \\
Cladosporium & 28.7 & 31.3 & 25.5 \\
Stemphilium & 21.7 & 20.3 & 23.5 \\
Botrytis & 20 & 20.3 & 19.6 \\
Trichophyton & 20 & 15.6 & 25.5 \\
Alternaria & 18.3 & 15.6 & 21.6 \\
Curvularia & 18.3 & 18.8 & 17.6 \\
Fusarium & 17.4 & 12.5 & 23.5 \\
Mucor & 15.7 & 17.2 & 13.7 \\
Rhizopus & 15.6 & 18.8 & 13.7 \\
Aureubasidium & 14.8 & 12.5 & 17.6 \\
Aspergillus Niger & 13.9 & 17.2 & 11.8 \\
Aspergillus fumigatus & 13.9 & 15.6 & 11.8 \\
Penicillium & 7.8 & 4.7 & 11.8 \\
Helmintosporium & 4.35 & 4.7 & 3.9 \\
Phoma & 2.65 & 1.6 & 3.9
\end{tabular}

media 4.3 y DE $=0.52$ ) y Trichophyton rubrum con $\mathrm{p}=0.023$ (niños mediana 3.25 y rango intercuartil 0.9 y adultos media $5.3 \mathrm{DE}=2.06$ )

El estudio encontró que la sensibilidad a hongos se asocia con sensibilidad a otros alérgenos. El $88.7 \%$ estaban sensibilizados a ácaros, $69.6 \%$ a epitelios, $40.9 \%$ a cucaracha, $20.9 \%$ a pólenes de árboles, $13 \%$ a pólenes de cereales, $28.7 \%$ a gramíneas, $34.8 \%$ a pólenes de hierbas, a mosquito $46.1 \%$ y a pólenes de cereales $13 \%$. El Cuadro 7 muestra también la sensibilidad a otros alérgenos en niños y adultos.

De los 115 pacientes evaluados, nueve solo tenían sensibilidad a hongos (7.8\%), sensibilidad a hongos y a un grupo más de alérgenos $6.1 \%$, sensibilidad a dos grupos más $10.4 \%$, sensibilidad a tres grupos más de alérgenos $29.6 \%$.

De los adultos asmáticos sensibilizados a hongos, 92.3\% estaban sensibilizados a epitelios y $30.7 \%$ a cucaracha, mientras que en los niños 65\% estaban sensibilizados a epitelios y $47.5 \%$ a cucaracha.
Cuadro 7. Distribución de sensibilidad a otros alérgenos en la población total, niños y adultos

\begin{tabular}{|c|c|c|c|}
\hline & Total & Niños & Adultos \\
\hline Sensibilidad a ácaros & 88.7 & 93.8 & 82.4 \\
\hline Der $p$ & 88.7 & 93.8 & 82.4 \\
\hline Der $f$ & 87 & 92.2 & 80.4 \\
\hline Blomia & 79.1 & 85.9 & 70.6 \\
\hline Sensibilidad polen de árboles & 20.9 & 14.1 & 29.4 \\
\hline Roble & 7 & 6.3 & 9.8 \\
\hline Abedul & 7 & 6.3 & 7.8 \\
\hline Ciprés & 13.9 & 6.3 & 23.5 \\
\hline $\begin{array}{l}\text { Sensibilidad al polen de } \\
\text { cereales }\end{array}$ & 13 & 12.5 & 13.7 \\
\hline Avena & 6.1 & 6.3 & 5.9 \\
\hline Cebada & 7.1 & 6.3 & 7.8 \\
\hline Centeno & 5.2 & 4.7 & 5.9 \\
\hline Trigo & 6.1 & 4.7 & 7.8 \\
\hline Maíz & 8.7 & 6.3 & 11.8 \\
\hline $\begin{array}{l}\text { Sensibilidad al polen de } \\
\text { gramíneas }\end{array}$ & 28.7 & 20.3 & 39.2 \\
\hline Dactilis glomerata & 6.1 & 3.1 & 9.8 \\
\hline Festuca elatior & 8.7 & 6.3 & 11.8 \\
\hline Lollium perenne & 11.3 & 6.3 & 17.6 \\
\hline Phleum pratense & 19.1 & 12.5 & 27.5 \\
\hline Poa & 13.9 & 9.4 & 19.6 \\
\hline Cynodon dactylon & 13 & 12.5 & 13.7 \\
\hline Phragmitis comunis & 10.4 & 7.8 & 13.7 \\
\hline $\begin{array}{l}\text { Sensibilidad al polen de } \\
\text { hierbas }\end{array}$ & 34.8 & 29.7 & 41.2 \\
\hline Artemisa vulgaris & 11.3 & 7.8 & 15.7 \\
\hline Chenopodium album & 20 & 17.2 & 23.5 \\
\hline Plantago lanceolata & 7.8 & 7.8 & 7.8 \\
\hline Salsola & 8.7 & 1.6 & 17.6 \\
\hline Parietaria judaica & 7 & 7.8 & 5.9 \\
\hline Taraxacum & 7 & 3.1 & 11.8 \\
\hline Ambrosia elatior & 6.1 & 3.1 & 9.8 \\
\hline Sensibilidad a epitelios & 69.6 & 65.6 & 74.5 \\
\hline Perro & 65.2 & 60.9 & 70.6 \\
\hline Gato & 28.7 & 23.4 & 35.3 \\
\hline Caballo & 23.5 & 15.6 & 33.3 \\
\hline Cucaracha & 40.9 & 48.4 & 31.4 \\
\hline Mosquito & 46.1 & 54.7 & 35.3 \\
\hline
\end{tabular}

\section{DISCUSIÓN}

En este estudio se encontró una sensibilidad a hongos en $12.8 \%$ de los pacientes a los que se les realizó prueba cutánea (prick) de alergias 
incluyendo 16 hongos, diferente a la encontrada en la IPS Universitaria en la misma ciudad con $5.4 \% .{ }^{29} \mathrm{El}$ estudio FRAAT de Cartagena demostró una sensibilidad de $3 \%,{ }^{30}$ el estudio de salud respiratoria de la Comunidad Europea encontró una sensibilización a Cladosporium de $1.7 \%$ y a Alternaria de $3.3 \% .{ }^{31}$ El estudio de Karakaya en Turquía encontró una sensibilidad a Alternaria inicial de $1.4 \%$ que al reevaluarla, en promedio 43.4 meses después, disminuía a $0.5 \%,{ }^{32}$ pero compatible con sensibilidades reportadas en otros estudios que varían entre 3 y $20 \%$ en Europa, $10 \%$ en Escandinavia, 30$60 \%$ en Estados Unidos ${ }^{13}$ y $6.7 \%$ en China, ${ }^{14}$ y $16.6 \%$ en Monterrey-México. ${ }^{23}$ Pocos estudios encontraron altos porcentajes de sensibilidad a hongos como el del servicio de Otorrinolaringología en Galveston Texas, donde Calhoun y colaboradores encontraron que los pacientes con pruebas cutáneas positivas tuvieron sensibilidad al menos a un hongo en $60 \% .{ }^{33}$ Una explicación a las variaciones en las sensibilidades puede deberse a que la exposición a estos es variable o a que se evalúa mayor o menor número de alérgenos de hongos.

Medellín no tiene estaciones y no existen estudios de aerobiología en la ciudad que determinen la concentración de esporas en el ambiente, por lo que el porcentaje de sensibilización alto a hongos no podría explicarse por la época del año en que fueron realizadas las pruebas de prick. Además, las pruebas se tomaron durante todos los meses.

La sensibilidad fue más frecuente a Candida seguida por Cladosporium, Stemphilium, Botrytis y Trichophyton. Esto es similar a lo encontrado en Caracas, Venezuela, donde la principal sensibilidad a hongos fue a Neurospora sitophila, seguida de Candida albicans ${ }^{19}$ y, a diferencia de lo encontrado en otros estudios donde los hongos más prevalentes son Alternaria alternata, Aspergillus fumigatus y Cladosporium. ${ }^{8,9,29,31}$
Los pacientes de este estudio tuvieron sensibilidad aislada a hongos en $7.8 \%$ mientras que $29.6 \%$ tuvo sensibilidad a hongos asociada con tres grupos más de alérgenos. Estuvieron sensibilizados a un hongo en $52.2 \%$ de los casos y más de tres hongos en $32.2 \%$. Los pacientes con sensibilidad a hongos tuvieron sensibilidad a otros alérgenos siendo los ácaros los más frecuentemente asociados, seguidos de los epitelios, cucaracha y los pólenes de hierbas. Esta polisensibilización se había detectado en $76.9 \%$ para la ciudad de Medellín ${ }^{29}$ y también se había reportado en países como Finlandia. ${ }^{9}$ La relación entre sensibilidad a hongos y sensibilidad a otros alérgenos se demostró en el estudio de Nolles donde se encontró que en niños de 0 a 15 años con asma o rinitis o eccema con IgE específicas previas a otros alérgenos, la sensibilidad a hongos era positiva en $18.2 \%$ para Cladosporium, $15.5 \%$ para Aspergillus, $14.6 \%$ para Alternaria y $7.3 \%$ para Penicillium y la probabilidad de estar sensibilizado a hongos se asoció con la IgE frente a perro, gato, árboles y grama. ${ }^{9}$

Las enfermedades más asociadas con la sensibilización a hongos en nuestro estudio fueron rinitis con $100 \%$, conjuntivitis con $92.2 \%$, asma $46.1 \%$, dermatitis atópica $20 \%$, al igual que lo encontrado en Finlandia por Reijula y colaboradores quienes descubrieron que la clínica presentada por los pacientes sensibilizados a hongos era asma en $44 \%$, dermatitis $58 \%$, rinitis $31 \%{ }^{8}$ El estudio FRAAT de Cartagena demostró que $100 \%$ de los individuos sensibilizados a hongos tenían síntomas de alergia. ${ }^{31}$ Estudios previos de prevalencia de enfermedades alérgicas realizados en Colombia, pero en población general, han encontrado prevalencia de síntomas de alergia más bajos a los reportados por nosotros. Es así como la prevalencia de asma en los últimos 12 meses fue del $10.4 \%$, de rinitis de $22.6 \%$, dermatitis atópica $3.9 \%$ y en Medellín la prevalencia encontrada fue de $13 \%$ en asma y de $28.2 \%$ en rinitis. ${ }^{24}$ Posteriormente, en los 
años 2009 y 2010 se efectuó otro estudio cuyos resultados sugieren que la prevalencia de síntomas de asma y rinitis estaba en aumento (asma de 10 a $12 \%$ y rinitis 23 vs $32 \%$ ). ${ }^{25}$ Bogotá tiene una prevalencia de rinoconjuntivitis en niños de 6 a 7 años de $17.2 \%$ y en adolescentes de 13 a 14 de $24.9 \% .^{34}$

En nuestro estudio la rinitis se encontró en 100\% de los pacientes sensibilizados a hongos y esta era persistente moderada a grave en $93.8 \%$ en los niños. Esta severidad de la rinitis también se asoció en Venezuela con la concentración de hongos del ambiente. ${ }^{19}$

El patrón multisistémico se presentó en un porcentaje cercano al $10 \%$ y se asoció con ser niño y la sensibilidad a Aspergillus fumigatus con un riesgo de $4.381($ IC95\% $=1.116-17.204)$ $p=0.024$. Otro estudio efectuado en Medellín mostró que los pacientes sensibilizados a hongos tenían un riesgo de patrón multisistémico de $4.9(1.7-13.7)$ y $\mathrm{p}<0.01$ comparado con los no sensibilizados a hongos. ${ }^{29}$ Este patrón multisistémico asociado con la sensibilización a hongos se propuso en ese estudio pero no se había reportado en ningún otro estudio llevado a cabo en Colombia, comprobando nuestro estudio la asociación entre estas dos variables, pero siendo Aspergillus fumigatus el responsable de la asociación.

La relación entre sensibilización a hongos y severidad de los síntomas de alergia se demostró en el estudio realizado en Salford Manchester por Ronan O Driscoll y colaboradores en donde evaluaron a 181 asmáticos con alérgenos de hongos y se encontró que de los asmáticos con más de una hospitalización por asma, 76\% tenían sensibilización a hongos comparados con $16 \%$ de asmáticos sin hospitalizaciones, con $\mathrm{p}<0.0001$ y tenían más de un hongo positivo en $50 \%$ comparados con $5 \%$ en asmáticos sin hospitalizaciones con $\mathrm{p}<0.0001$. El número de hospitalizaciones por asma se relacionó con el número y tamaño de alérgenos positivos con un coeficiente de correlación de Spearman de 0.6 mientras que para alérgenos no hongos el coeficiente de correlación fue de 0.34 . El estudio sugirió que $6 \%$ de las hospitalizaciones por asma en adultos y $19 \%$ de las hospitalizaciones por asma en edades comprendidas entre 16 y 40 años pueden satribuirse a alergia a hongos. ${ }^{16}$ En Turquía, Ceylan y colaboradores encontraron que los pacientes asmáticos con altas concentraciones de hongos en las casas estaban más sensibilizados a hongos y usaban más dosis de esteroides al compararlos con los no sensibilizados a hongos. ${ }^{4}$

El patrón mutisistémico no se relacionó en este estudio con las condiciones ambientales.

A pesar de no llegar a la significación estadística, los niños tuvieron más rinitis persistente moderada a grave, más alergia a medicamentos y más urticaria aguda que los adultos, mientras estos últimos eran más mujeres, tenían más alergia a alimentos y más urticaria crónica. Sí hubo asociación estadística entre ser niño y tener asma o dermatitis y estar sensibilizado a hongos.

En los pacientes sensibilizados a hongos de este estudio, ni el asma ni el patrón multisistémico se asociaron con las condiciones ambientales a las que estaban expuestos los pacientes.

\section{CONCLUSIONES}

El patrón de sensibilidad a hongos mostró que los más frecuentemente positivos fueron Candida, Cladosporium, Stemphilium, Botrytis y Trichophyton. La sensibilidad aislada a hongos es poco frecuente y se asocia con sensibilidad a otros alérgenos, como ácaros, epitelios, hierbas o gramíneas en ese orden. La mayoría de los pacientes está polisensibilizada. El patrón multisistémico con rinitis, conjuntivitis, asma 
y dermatitis se presentó más en niños y se relacionó con sensibilidad a Aspergillus. Una tercera parte de los pacientes sensibilizados a hongos tuvo sensibilidad a más de tres hongos y el tamaño de las pápulas fue muy cercano al límite de positividad.

Por los hallazgos de este estudio, los alérgenos de hongos poco evaluados en Colombia como Candida y Cladosporium deberían incluirse en los paneles de neumoalérgenos que utilizan los alergólogos. Debería descartarse la sensibilidad a Aspergillus fumigatus en niños con patrón multisistémico.

\section{Agradecimientos}

A la docente de epidemiología de la Universidad CES, Gloria Sierra por su colaboración en estadística.

Los autores declaran que no tienen conflictos de interés en la publicación de este estudio.

\section{REFERENCIAS}

1. Kauffman HF, van der Heide S. Exposure, sensitization, and mechanisms of fungus-induced asthma. Curr Allergy Asthma Rep. 2003;3(5):430-7.

2. Barnes CS, Dowling P, Van Osdol T, Portnoy J. Comparison of indoor fungal spore levels before and after professional home remediation. Ann Allergy Asthma Immunol Off Publ Am Coll Allergy Asthma Immunol. 2007;98(3):262-8.

3. Eggleston PA. Environmental control for fungal allergen exposure. Curr Allergy Asthma Rep. 2003;3(5):424-9.

4. Ceylan E, Ozkutuk A, Ergor G, Yucesoy M, Itil O, Caymaz $S$, et al. Fungi and indoor conditions in asthma patients. J Asthma Off J Assoc Care Asthma. 2006;43(10):789-94.

5. Dziadzio L, Bush RK. Assessment and control of fungal allergens. Curr Allergy Asthma Rep. 2001;1(5):455-60.

6. Quansah R, Jaakkola MS, Hugg TT, Heikkinen SAM, Jaakkola JJK. Residential dampness and molds and the risk of developing asthma: a systematic review and meta-analysis. PloS One. 2012;7(11):e47526.

7. Jaakkola MS, Quansah R, Hugg TT, Heikkinen SAM, Jaakkola JJK. Association of indoor dampness and molds with rhinitis risk: a systematic review and meta-analysis. J Allergy Clin Immunol. 2013;132(5):1099-110.e18.
8. Reijula K, Leino $M$, Mussalo-Rauhamaa $H$, Nikulin $M$, Alenius $\mathrm{H}$, Mikkola J, et al. IgE-mediated allergy to fungal allergens in Finland with special reference to Alternaria alternata and Cladosporium herbarum. Ann Allergy Asthma Immunol Off Publ Am Coll Allergy Asthma Immunol. 2003;91(3):280-7.

9. Nolles G, Hoekstra MO, Schouten JP, Gerritsen J, Kauffman $H F$. Prevalence of immunoglobulin $E$ for fungi in atopic children. Clin Exp Allergy J Br Soc Allergy Clin Immunol. 2001;31(10):1564-70.

10. Değer L, Plante $C$, Goudreau S, Smargiassi A, Perron S, Thivierge RL, et al. Home environmental factors associated with poor asthma control in Montreal children: a population-based study. J Asthma Off J Assoc Care Asthma. 2010;47(5):513-20.

11. Schram D, Doekes G, Boeve M, Douwes J, Riedler J, Ublagger $E$, et al. Bacterial and fungal components in house dust of farm children, Rudolf Steiner school children and reference children--the PARSIFAL Study. Allergy. 2005;60(5):611-8.

12. Alfvén $T$, Braun-Fahrländer $C$, Brunekreef $B$, von Mutius $E$, Riedler J, Scheynius A, et al. Allergic diseases and atopic sensitization in children related to farming and anthroposophic lifestyle--the PARSIFAL study. Allergy. 2006;61(4):414-21.

13. D'Amato $G$, Chatzigeorgiou G, Corsico R, Gioulekas D, Jäger $L$, Jäger $S$, et al. Evaluation of the prevalence of skin prick test positivity to Alternaria and Cladosporium in patients with suspected respiratory allergy. A European multicenter study promoted by the Subcommittee on Aerobiology and Environmental Aspects of Inhalant Allergens of the European Academy of Allergology and Clinical Immunology. Allergy. 1997;52(7):711-6.

14. Lin J, Su N, Liu G, Yin K, Zhou X, Shen H, et al. The impact of concomitant allergic rhinitis on asthma control: a crosssectional nationwide survey in China. J Asthma Off J Assoc Care Asthma. 2014;51(1):34-43.

15. Zureik M, Neukirch C, Leynaert B, Liard R, Bousquet J, Neukirch F, et al. Sensitisation to airborne moulds and severity of asthma: cross sectional study from European Community respiratory health survey. BMJ. 2002;325(7361):411-4.

16. O'Driscoll BR, Hopkinson LC, Denning DW. Mold sensitization is common amongst patients with severe asthma requiring multiple hospital admissions. BMC Pulm Med. 2005;5:4.

17. Agarwal R. Severe asthma with fungal sensitization. Curr Allergy Asthma Rep. 2011;11(5):403-13.

18. Vicencio AG, Santiago MT, Tsirilakis K, Stone A, Worgall S, Foley EA, et al. Fungal sensitization in childhood persistent asthma is associated with disease severity. Pediatr Pulmonol. 2014;49(1):8-14.

19. Galante D, Hartung de Capriles C, Mata-Essayag S, Conesa A, Córdova Y, Trejo E, et al. Respiratory allergies in Venezuela: are fungi responsible? Mycoses. 2006;49(6):493-8.

20. Niemeijer NR, de Monchy JG. Age-dependency of sensitization to aero-allergens in asthmatics. Allergy. 1992;47(4 Pt 2):431-5. 
21. Arbes SJ, Gergen PJ, Elliott L, Zeldin DC. Prevalences of positive skin test responses to 10 common allergens in the US population: results from the third National Health and Nutrition Examination Survey. J Allergy Clin Immunol. 2005;116(2):377-83.

22. Salo PM, Arbes SJ, Jaramillo R, Calatroni A, Weir $\mathrm{CH}$, Sever $\mathrm{ML}$, et al. Prevalence of allergic sensitization in the United States: results from the National Health and Nutrition Examination Survey (NHANES) 2005-2006. J Allergy Clin Immunol. 2014;134(2):350-9.

23. Elizondo-Villarreal, Barbara, Gonzalez-Diaz;Sandra, AriasCruz, Alfredo, Leal-Villarreal, Lucia, Del Carmen Zarate-Hernandez, Maria, Rivero-Arias, Dulce $M$, et al. Prevalence of Sensitization to Mold Allergens in Patients with Respiratory Allergy. J Allergy Clin Immunol. 2016;137(2):AB30.

24. Dennis R, Caraballo L, García E, Caballero A, Aristizabal G, Córdoba $\mathrm{H}$, et al. Asthma and other allergic conditions in Colombia: a study in 6 cities. Ann Allergy Asthma Immunol Off Publ Am Coll Allergy Asthma Immunol. 2004;93(6):56874.

25. Dennis RJ, Caraballo L, García E, Rojas MX, Rondon MA, Pérez $A$, et al. Prevalence of asthma and other allergic conditions in Colombia 2009-2010: a cross-sectional study. BMC Pulm Med. 2012;12:17.

26. Rodriguez Laura Andrea, Rey Juan Jose, Herrera Astrid Berena, Castro Henry, Vera Lina Maria, Cala Luz Libia, et al. Prevalencia de síntomas respiratorios indicativos de asma y asociación con contaminación atmosférica en preescolares de Bucaramanga, Colombia. Biomedica. 30(1):15-22.

27. Herrera, Astrid, Rodriguez, Laura, Niederbacher,Jürg. Contaminación biológica intradomiciliaria y su relación con síntomas respiratorios indicativos de asma bronquial en preescolares de Bucaramanga, Colombia. Biomedica. 2011;31:357-71.

28. Quiroz Arcentales, leonardo, Hernandez Florez, Luis, Agudelo CAlderón, Carlos, Medina, Katalina, Robledo MArtinez, Rocio, Osorio GArcia, Samuel. Enfermedad y síntomas respiratorios en cinco municipios carboníferos del Cesar, Colombia. Rev Salud Pública. 2013;15(1):66-79.

29. Sánchez-Caraballo J, Diez-Zuluaga S, Cardona-Villa R. [Sensitization to aeroallergens in allergic patients from Medellin, Colombia]. Rev Alerg Mex Tecamachalco Puebla Mex 1993. septiembre de 2012;59(3):139-47.

30. Acevedo N, Sánchez J, Zakzuk J, Bornacelly A, Quiróz C, Alvarez Á, et al. Particular characteristics of allergic symptoms in tropical environments: follow up to 24 months in the FRAAT birth cohort study. BMC Pulm Med. 2012;12:13.

31. Bousquet P-J, Chinn S, Janson C, Kogevinas M, Burney $P$, Jarvis $D$, et al. Geographical variation in the prevalence of positive skin tests to environmental aeroallergens in the European Community Respiratory Health Survey I. Allergy. 2007;62(3):301-9.

32. Karakaya G, Kalyoncu AF. The natural course of atopy determined by skin prick tests in patients with bronchial asthma and/or rhinitis. Allergol Immunopathol (Madr). 2006;34(6):257-62.

33. Calhoun KH. Patterns of mold sensitivity in the subtropical Gulf Coast. Otolaryngol--Head Neck Surg Off J Am Acad Otolaryngol-Head Neck Surg. 2004;130(3):306-11.

34. Peñaranda A, Aristizabal G, García E, Vásquez C, RodríguezMartinez CE. Rhinoconjunctivitis prevalence and associated factors in school children aged 6-7 and 13-14 years old in Bogota, Colombia. Int J Pediatr Otorhinolaryngol. 2012;76(4):530-5. 